\title{
The effects of increased oily fish intake during pregnancy on neonatal immune cells - results from the salmon in pregnancy study (SIPS)
}

\author{
P. S. Noakes, M. Vlachava, L.-S. Kremmyda, N. D. Diaper, E. A. Miles and P. C. Calder \\ Institute of Human Nutrition and DOHaD Division, School of Medicine, University of Southampton, IDS Building, \\ Southampton General Hospital, Southampton SO16 6YD, UK
}

The development of childhood allergic disease is frequently preceded by immunologic differences that are most evident in the neonatal period $^{(1)}$. Preliminary studies also suggest that maternal environmental exposures (such as diet) can modify neonatal T-cell function, although the mechanisms are not clear ${ }^{(2)}$. With rising allergic disease rates, there is a continuing urgency to identify the pathways involved and to explore the effects of early interventions that could favourably influence the functional development of T-cell responses and prevent allergic disease. Dietary $n-3$ polyunsaturated fatty acids (PUFA), found in oily fish and in fish oils, may represent a mode of allergy prevention. Fish oil supplementation during pregnancy alters neonatal immunity in a way that would be consistent with lowered risk of atopy ${ }^{(3)}$. However, there are no studies of the influence of increased oily fish consumption in pregnancy on neonatal immune cell phenotypes.

At 20 weeks gestation, 123 allergic, pregnant women with low habitual intake of oily fish $(\leq 2 /$ month) were randomised to consume two portions of salmon per week (each portion of salmon provided about $2 \mathrm{~g}$ of $n-3$ PUFA) or to continue their habitual diet of low oily fish consumption until delivery. Mononuclear cells were purified from umbilical cord blood $(n=101)$ and the percentages of T-regulatory cells (defined as $\mathrm{CD} 4{ }^{+} \mathrm{CD} 25^{+} \mathrm{CD} 127^{\mathrm{lo} /-}$ cells) and monocytes (defined as CD $14^{+} \mathrm{TLR}-2^{+}$cells) were examined by flow cytometry following staining the cells with relevant fluorescently labelled antibodies. Percentages of cord blood T-regulatory cells and monocytes were not different between the control and oily fish intervention groups $(P=0.727$ and $P=0.364$, respectively). Similarly, surface expression of $\mathrm{CD} 25^{+} \mathrm{CD} 127^{\mathrm{lo} /-}$ on $\mathrm{CD} 4{ }^{+}$T-cells (mean fluorescence intensity) was not different between the two groups $(P=0.159)$.

In conclusion, oily fish intervention in pregnancy does not alter numbers of neonatal regulatory T-cells or TLR-2 expressing monocytes. Further assessment of cellular immune function and clinical follow-up of these infants will determine if there are any significant effects of maternal oily fish intake on postnatal immune development and expression of allergic disease.

This work was supported by the European Commission under Framework 6 and forms part of the AquaMax integrated project (FOOD-CT-2006-016249-2). The authors wish to thank staff at the Princess Anne Hospital, Southampton and the Medical Research Council Epidemiology Resource Centre for their invaluable contributions to this study.

1. Prescott S, Macaubas C, Smallacombe T et al. (1999) Lancet 353, 196-200.

2. Willers S, Devereux G, Craig L et al. (2007) Thorax 62, 773-779.

3. Dunstan JA, Mori TA, Bardent A et al. (2003) Clin Exp Allergy 33, 442-448. 